\title{
Lois et règlements au Journal Officiel
}

Arrêtés du 25 avril 1995 modifiant l'arrêté du 3 avril 1985 fixant la Nomenclature des actes de biologie médicale

\author{
(J.O. du 28 avril 1995, 6564-6565)
}

Art.1er - A la deuxième partie de la Nomenclature des actes de biologie médicale, le chapitre 4 est annulé et remplacé par le chapitre suivant :

\section{Spermiologie}

\section{I - EXAMENS DE BASE}

$\mathrm{n}^{\circ} 0070$ - Spermogramme et spermocytogramme.....B110

Cet examen comporte : volume de l'éjaculat, $\mathrm{pH}$ (papier), estimation de la viscosité, numération des spermatozoïdes et des cellules rondes, éventuellement des leucocytes, évaluation du pourcentage des spermatozoïdes mobiles (progressifs et non progressifs), dans la première heure et après trois heures, pourcentage des spermatozoïdes vivants dans la première heure, présence ou non d'une agglutination spontanée et caractères des agglutinats, compte sur 100 spermatozoïdes des différentes anomalies de la tête et du flagelle, établissement de l'index d'anomalies multiples, compte pour 100 spermatozoïdes des cellules de la lignée spermatique, des autres cellules et des flagelles isolés.

$n^{\circ} 0071$ - Test-post coïtal ou test de Huhner (TPC).....B45

Le résultat doit comporter : le jour du cycle, le délai depuis le coït, le degré de dilatation du col, de l'abondance, de la filance et de la transparence de la glaire; la densité de spermatozoïdes par champ et le pourcentage de spermatozoïdes mobiles progressifs, non progressifs (avec précision du caractère éventuellement oscillant) et immobiles.

Lorsque les examens $n^{\circ} 0070$ et $n^{\circ} 0071$ ne mettent pas en évidence d'anomalies, il y a lieu de considérer qu'il n'y a pas de participation masculine à la stérilité du couple et donc de ne pas aller plus loin dans l'exploration du sperme. Dans ce cas, les examens «épreuves complémentaires»inscrits cidessous ne peuvent donner lieu à cotation.

\section{II - EPREUVES COMPLEMENTAIRES}

Les examens ci-dessous doivent être explicitement prescrits. Ils sont soumis aux dispositions de l'article 5 des dispositions générales. La demande d'entente préalable doit être accompagnée des résultats des examens $n^{\circ} 0070$ et 0071 . 
Le compte rendu des épreuves complémentaires doit rappeler les anomalies qui ont conduit à les pratiquer. Les épreuves complémentaires peuvent être cotées dans les conditions suivantes :

1 - Les examens de bases sont profondément perturbés (TPC négatif, azoospermie ou asthénozoospermie totale) : exploration bio-chimique de la fonction prostato-vésiculaire $\left(n^{\circ} 0072\right)$ et épididymaire $\left(n^{\circ} 0073\right)$, éventuellement spermoculture (la cotation applicable est, dans ce cas, celle de l'examen $n^{\circ} 0204$ );

2 - Les examens de base révèlent un déficit important mais non profond (TPC médiocre ou oligozoospermie, asthénozoospermie, tératozoospermie isolées ou associées) : étude de l'éjaculat fractionné ( $\left.n^{\circ} 0074\right)$, test de migration survie des spermatozoïdes $\left(n^{\circ} 0075\right)$ et éventuellement spermoculture $\left(n^{\circ} 0204\right)$, explorations biochimiques de la fonction épididymaire ( $\left.n^{\circ} 0073\right)$, recherche d'immunisation masculine ( $n^{\circ} 0076$ et, le cas échéant, $\left.n^{\circ} 0077\right)$;

3- Les examens de base révèlent une discordance de résultats (TPC médiocre ou nul alors que les caractéristiques du sperme sont normales ou peu perturbées) : test de pénétration croisée in vitro $\left(n^{\circ} 0078\right)$, recherche d'une immunisation masculine $\left(n^{\circ} 0076\right.$ et éventuellement, $\left.n^{\circ} 0077\right)$ et $/$ ou féminine $\left(n^{\circ} 0079\right)$;

4 - Anéjaculation ou volume de sperme anormalement faible : recherche d'une éjaculation rétrograde $\left(n^{\circ} 0080\right)$;

5 - Echecs répétés de F.I.V. avec le sperme du conjoint après trois cycles marqués par un taux de clivage nul ou très faible (inférieur à 30 p.100) : avant toute nouvelle tentative, recherche d'immunisation masculine ( ${ }^{\circ} 0076$ et, éventuellement $\left.n^{\circ} 0077\right)$, coloration des spermatozoïdes au bleu d'aniline $\left(n^{\circ} 0081\right)$.

$n^{\circ} 0072$ - Exploration biochimique de la fonction prostato-vésiculaire...B175 comportant :

-dosage du fructose séminal (technique enzymatique);

-deux des trois dosages suivants :

-citrate séminal (technique enzymatique) ;

-phosphatase acide séminale ;

-zinc séminal.

$\mathrm{n}^{\circ} 0073$ - Exploration de la fonction épididymaire......B85

comportant :

-dosage de la carnitine libre séminale (technique enzymatique)

ou

-dosage de l'apha 1-4 glucosidase séminale (technique enzymatique)

Le résultat doit mentionner le volume de l'éjaculat.

$n^{\circ} 0074$ - Etude de l'éjaculat fractioné pour exploration d'une oligospermie confirmée.....B130 
La technique doit comporter une recherche de fructose et une numération des spermatozoïdes dans les deux fractions. La fraction riche en spermatozoïdes donne lieu aux mêmes mesures et évaluations que l'examen $n^{\circ} 0070$.

\section{$n^{\circ} 0075$ - Test de migration-survie des spermatozoïdes ....B120}

comportant isolement des spermatozoïdes mobiles dans un milieu de fécondation in vitro (quelle que soit la technique, y compris par centrifugation en gradient de Percoll), numération du pourcentage de spermatozoïdes mobiles progressifs et non progressifs, avant, après migration et après un délai de six heures minimun et établissement de la morphologie avant et après migration.

Acte diagnostique non cumulable avec l'acte 0051 du chapitre PMA.

\section{Recherche d'une immunisation anti-spermatozoïdes chez l'homme :}

$\mathbf{n}^{\circ} 0076$ - a) Recherche d'anticorps fixés sur les spermatozoïdes.....B50 :

-par réaction d'agglutination mixte (MAR-test) utilisant des hématies humaines Rh positif revêtues d'IgG anti Rh

ou

-par technique utilisant des immunobilles revêtues d'antiglobulines anti Ig humaines.

$n^{\circ} 0077$-b) Recherches indirectes d'anticorps antispermatozoïdes dans le sérum sanguin et simultanément dans le plasma séminal. Les deux recherches : B280

comportant un titrage des anticorps par la technique des immunobilles.

Le résultat (des examens $\mathrm{n}^{\circ} 0076$ et 0077 ) doit mentionner le titre des anticorps, leur type (IgG, IgA, IgM) et leur topographie de fixation sur les spermatozoïdes.

L'examen $\mathrm{n}^{\circ} 0077$ ne peut être coté qu'en cas de résultat positif de l'examen $\quad \mathrm{n}^{\circ} 0076$.

$n^{\circ} 0078$ - Pénétration du mucus cervical, test croisé.....B120

comportant la pénétration des spermatozoïdes testés et spermatozoïdes témoins dans le mucus cervical à tester et dans le mucus cervical humain témoin.

Le résultat doit mentionner : pour la patiente, le jour du cycle et la dilatation du col ; pour le mucus à tester, l'abondance, la filance et la transparence et pour les spermes (témoins et à tester), la numération et la mobilité des spermatozoïdes.

Il doit comporter des données sur la pénétration (rapidité, densité, distance parcourue...) et la survie des spermatozoïdes (notamment mobilité à six heures avec caractéristiques).

$n^{\circ} 0079$ - Recherche d'une immunisation antispermatozoïdes chez la femme par recherches indirectes d'anticorps anti-spermatozoïdes dans le sérum sanguin et simultanément dans le mucus cervical. Les deux recherches : B280

comportant un titrage des anticorps par la technique des immunobilles.

Le résultat doit comporter les mêmes mentions que pour l'examen $n^{\circ} 0077$.

$n^{\circ} 0080$ - Etude d'une éjaculation rétrograde.....B110

en cas d'anéjaculation ou d'éjaculation de faible volume (inférieur à $1 \mathrm{ml}$ ) par réalisation du spermogramme et spermocytogramme sur premier jet d'urine après 
masturbation.

Le résultat doit comporter les mêmes indications que pour l'examen $n^{\circ} 0070$ et mentionner le volume du premier jet d'urine.

\section{$\mathbf{n}^{\circ} 0081$ - Coloration des spermatozoïdes au bleu d'aniline.....B50} en cas de pourcentage d'anomalies de la tête au spermocytogramme supérieur à 80 p.100 ou d'échecs répétés d'A.M.P. avec sperme du conjoint. 


\section{Décret $\mathrm{n}^{\circ}$ 95-558 du 6 mai 1995 relatif à la Commission nationale de méde- cine et biologie de la reproduction et du diagnostic prénatal et modifiant le code de la santé publique (deuxième partie : Décrets en Conseil d'Etat). (J.O. du 7 mai 1995, 7360-7361)}

Art.1er - Il est inséré dans la section IV du chapitre V du titre Ier du livre II du code de la santé publique (deuxième partie : Décrets en Conseil d'Etat) une sous-section II ainsi rédigée :

«Sous-section II»

«Commission nationale de médecine et de biologie de la reproduction et du diagnostic prénatal

«Paragraphe Ier

«Composition et organisation de la commission

«Art.R. 183-4-1. - La Commission nationale de médecine et de biologie de la reproduction et du diagnostic prénatal instituée à l'article L. 184-3 comprend, outre son président, des membres de droit et des membres nommés par arrêté du ministre chargé de la santé.

«Art.R. 184-3-2. - Le mandat des membres nommés est de trois ans. Il est renouvelable.

«Art.R. 184-3-3" - La commission comprend deux sections : la section de l'assistance médicale à la procréation et la section du diagnostic prénatal.

«La formation plénière est composée de l'ensemble des membres de la commission.

«Art.R. 184-3-4. - Sont membres de droit de chacune des deux sections :

" $1^{\circ}$ Le directeur général de la santé ou son représentant;

" $2^{\circ}$ Le directeur des hôpitaux ou son représentant ;

$\ll 3^{\circ}$ Le directeur de la sécurité sociale ou son représentant ;

«4 $4^{\circ}$ Le directeur général de la recherche et de la technologie au ministère chargé

de la recherche ou son représentant ;

« $5^{\circ}$ Le directeur des affaires civiles et du sceau au ministère de la justice ou son représentant;

$« 6^{\circ}$ Le président du Conseil national de l'ordre des médecins ou son représentant;

«7 $7^{\circ}$ Le président du conseil central de la section G de l'ordre des pharmaciens ou son représentant ;

$« 8^{\circ}$ Le directeur de la Caisse nationale d'assurance maladie des travailleurs salariés ou son représentant;

$« 9^{\circ}$ Le directeur de l'Institut national de la santé et de la recherche médicale ou son représentant.

«Art.R. 184-3-5. - Sont nommés par arrêté du ministre chargé de la santé :

«I. membres de chacune des deux sections :

«a) Un représentant du Comité consultatif national d'éthique pour les sciences de la vie et de la santé, proposé par le président du comité ;

«b) Un représentant des associations familiales, choisi sur une liste de trois personnes établie par le président de l'Union nationale des associations familiales ;

«c) Un médecin inspecteur d'une direction régionale ou départementale des afffaires sanitaires et sociales ;

«) Un pharmacien inspecteur d'une direction régionale des affaires sanitaires et sociales ;

«e) Une haute personnalité scientifique ; 
«f) Un spécialiste du droit de la filiation ;

«g) Un praticien ayant une formation ou une expérience particulière en génétique humaine, choisi sur une liste de trois personnes établie par la Société française de génétique humaine.

«2. Membres de la section de l'assistance médicale à la procréation.

«A. - Praticiens désignés sur proposition des organisations représentatives :

«a) Un gynécologue-obstétricien et un biologiste, choisis sur une liste de trois gynécologues-obstétriciens et de trois biologistes établie par le Groupe d'étude de la fécondation in vitro en France ;

«b) Un biologiste de la reproduction, choisi sur une liste de trois biologistes établie par la Fédération des biologistes des laboratoires d'études de la fécondation et de la conservation de l'œuf ;

«c) Deux praticiens, l'un clinicien et l'autre biologiste, choisis sur une liste de six personnes établie par la Fédération des centres d'études et de conservation des oufs et du sperme humains ;

«B. - Personnalités compétentes :

«d) Un médecin choisi en raison de sa compétence dans le domaine de l'assistance médicale à la procréation avec tiers donneur ;

«e) Un épidémiologiste ayant une expérience en médecine de la reproduction;

(f) Un gynécologue-obstétricien et un biologiste d'un établissement public de santé ayant une expérience dans le domaine de l'assistance médicale à la procréation ;

«g) Un gynécologue-obstétricien d'un établissement de santé privé ayant une expérience dans le domaine de l'assistance médicale à la procréation ;

«h) Un directeur de laboratoire d'analyses de biologie médicale ayant une expérience dans le domaine de l'assistance médicale à la procréation :

«i) Un médecin choisi en raison de son expérience en andrologie (souligné par la rédaction)

«j) Une personnalité scientifique choisie en raison de sa compétence dans la recherche en matière d'assistance médicale à la procréation.

«3. Membres de la section du diagnostic prénatal :

«A. - Praticiens désignés sur proposition des organisations représentatives :

«a) Deux praticiens ayant une expérience de diagnostic prénatal, choisis sur une liste de six personnes établie par l'Association française pour le dépistage et la prévention des handicaps de l'enfant;

«b) Un médecin choisi sur une liste de trois personnes établie par l'Association des cytogénéticiens de langue française ;

(c) Un gynécologue-obstétricien expérimenté en matière de prélèvements sur le foetus, choisi sur une liste de trois personnes établie par le collège national des gynécologues et obstétriciens français ;

«d) Un médecin choísi sur une liste de trois personnes établie par la Société francophone de médecine fœtale ;

«B. - Personnalités désignés en raison de leur compétence :

«e) Un pédiatre exerçant son activité en maternité ;

«f) Deux médecins expérimentés en échographie fœtale ;

«g) Deux biologistes ayant une expérience particulière dans la réalisation d'examens de biologie foetale, dont l'un en biologie moléculaire ;

«h) Deux praticiens ayant une expérience particulière dans la réalisation d'examens de cytogénétique, dont l'un exerce dans le secteur public et l'autre dans le secteur privé ; 
«i) Une personnalité scientifique choisie en raison de sa compétence dans la recherche en matière de diagnostic prénatal.

«Art.R. 194-3-6. - En cas de cessation de fonction d'un membre de la commission en cours de mandat pour quelque cause que ce soit, son remplacement s'effectue dans les mêmes conditions que sa nomination et pour la durée du mandat restant à accomplir.

"Art.R. 184-3-7. - Tout membre de la commission nommé par le ministre chargé de la santé qui est absent, sans motif légitime, à plus de trois séances consécutives de la formation plénière ou des sections peut être remplacé dans les conditions prévues à l'article R.184-3-6.

«Paragraphe 2

\section{«Attributions de la commission}

«Art.R. 184-3-8. - La section de l'assistance médicale à la procréation donne au ministre chargé de la santé des avis motivés sur :

«1. Les demandes d'agrément des praticiens sous la responsabilité desquels sont effectués les actes cliniques et biologiques d'assistance médicale à la procréation, en vertu de l'article L.152-9 ; ces avis tiennent compte, notamment, de la formation, de la compétence et de l'expérience des praticiens ;

«2. Les demandes d'autorisation mentionnées aux articles L.184-1 et L.673-5, présentées par les établissements, laboratoires et organismes en vue d'exercer les activités d'assistance médicale à la procréation ; ces avis tiennent compte, notamment, des locaux et de l'équipement des centres, de l'organisation des activités et, le cas échéant, du volume d'activités et de la qualité des résultats obtenus ;

«3. Les demandes de renouvellement des agréments et autorisations ; ces avis tiennent compte des résultats de l'évaluation des activités des praticiens et des établissements et laboratoires ;

«4. Les retraits d'agrément et d'autorisation.

«Art.R 184-3-9. - La section du diagnostic prénatal donne au ministre chargé de la santé des avis motivés sur :

«1. Les demandes d'autorisation d'exercice des activités de diagnostic prénatal ; ces avis tiennent compte, notamment, de la formation, de la compétence et de l'expérience des praticiens responsables, des locaux et de l'équipement des centres, de l'organisation des activités et, le cas échéant, du volume d'activités et de la qualité des résultats obtenus ;

«2. Les demandes d'agrément des centres de diagnostic prénatal pluridisciplinaires mentionnés à l'article L.162-16 ; cet avis tient compte notamment de la formation, de la compétence et de l'expérience des praticiens et des modalités de fonctionnement des centres;

«3. Les demandes de renouvellement des autorisations et agréments, en tenant compte des résultats de l'évaluation des activités des centres ;

«4. Les retraits d'autorisation et d'agrément.

«Art. R. 184-3-10. - La commission réunie en formation pleinière exerce les attributions suivantes :

« $1^{\circ}$ En application de l'article L. 152-8, elle examine les projets d'études sur embryons, qui ne peuvent être mis en œuvre, dans des conditions définies par décret en Conseil d'Etat, que sur son avis conforme. L'avis de la commission est émis au vu du rapport écrit présenté par un groupe technique désigné par le président et composé d'au moins six membres appartenant pour moitié à chacune des deux sections ; 
« $2^{\circ}$ En application de l'article L. 162-17, elle donne un avis motivé sur les demandes d'autorisation de pratiquer les activités de diagnostic biologique à partir de cellules prélevées sur l'embryon in vitro ;

« $3^{\circ}$ Elle donne les avis mentionnés aux articles R.184-3-8 et R.184-3-9 lorsque l'affaire est renvoyé devant la formation plénière par le président de la commission, d'office ou à la demande de la majorité des membres d'une des deux sections ;

« $4^{\circ}$ Elle adopte le rapport annuel prévu au deuxième alinéa de l'article L. 184-3.

«Art. R.184-3-11. - Le rapport annuel mentionné à l'article précédent comporte un bilan des travaux de la commission et notamment de ses avis sur les demandes d'autorisation et d'agrément. Il présente l'évolution, pendant l'année écoulée, de la médecine et de la biologie de la reproduction et du diagnostic prénatal, et fait apparaître les avancées scientifiques et techniques ainsi que leurs enjeux.

«Il comporte des recommandations tendant à promouvoir la qualité des activités exercées et à améliorer leur évaluation ainsi que des propositions en matière d'enseignement, d'information du public et de recherche, en particulier dans le domaine de l'épidémiologie.

«Pour l'élaboration de ce rapport, la commission a connaissance de la synthèse nationale des rapports annuels d'activité mentionnés aux articles L.184-2 et L.673-5.

"Art. R. 184-3-12. - Chacune des sections ou la commission siégeant en formation plénière donne son avis sur les questions relatives à la médecine et à la biologie de la reproduction et au diagnostic prénatal dont elle est saisie par le ministre chargé de la santé.

«Art. R. 184-3-13. - Le ministre chargé de la santé prend les mesures appropriées en vue de faire participer la commission au suivi et à l'évaluation du fonctionnement des établissements et laboratoires autorisés à exercer des activités d'assistance médicale à la procréation ou de diagnostic prénatal.

"La commission a communication des rapports annuels d'activité prévus aux articles L. 184-2 et L. 673-5 et participe à leur analyse. Elle peut formuler des recommandations et, si nécessaire, proposer des contrôles.

«Chaque section apporte un conseil scientifique et technique aux autorités administratives chargées du contrôle et de l'évaluation des établissements et laboratoires susmentionnés.

«Art. R. 184-3-14. - Chacune des sections ou la commission siégeant en formation plénière peut appeler l'attention du ministre chargé de la santé sur toute question relative à ses domaines de compétence.

\section{«Paragraphe 3}

\section{«Fonctionnement}

«Art. R. 184-3-15. - La commission en formation plénière ou chacune des sections se réunit sur convocation du président. Cette convocation est de droit si elle est demandée par le ministre chargé de la santé. La commission peut également être convoquée à la demande de la majorité de ses membres.

«Art. R. 184-3-16.- Le président préside les séances de la commission en formation plénière et les séances de chacune des deux sections.

"Le décret qui désigne le président prévoit celui qui, parmi les membres de droit, est appelé en son absence à le supléer dans ses fonctions.

«Art. R. 184-3-17. - La commission réunie en formation plénière ou chacune des sections ne peut se prononcer que si la moitié au moins de ses membres sont présents ; toutefois, quand la majorité requise n'est pas atteinte à une réunion, le même ordre du jour est 
reporté à un réunion ultérieure tenue dans un délai de quinze jours ; les délibérations prises lors de cette deuxième réunion sont valables quel que soit le nombre des membres présents.

«La commission réunie en formation plénière ou chacune des sections se prononce à la majorité des voix des membres présents ; en cas de partage égal des voix, celle du président est prépondérante.

«Art. R. 184-3-18. - Le président peut constituer des groupes de travail chargés de toute question soumise à la commission.

«Les rapports présentés à la commission peuvent être confiés par le président à des membres de la commission, à des membres de l'inspection générale des affaires sociales, à des fonctionnaires de l'administration centrale ou des services déconcentrés du ministre chargé de la santé.

«Art. R. 184-3-19. - Le président peut appeler à participer aux travaux de la commission ou des groupes de travail, à titre consultatif et pour une ou plusieurs séances, toute personne dont le concours lui paraît utile pour l'étude d'une question déterminée.

«Art. R. 184-3-20. - Le secrétariat de la commission est assuré par la direction générale de la santé."

Art. 2. - Le décret $\mathrm{n}^{\circ}$ 88-328 du 8 avril 1988 portant création de la Commission nationale de médecine et de biologie de la reproduction est abrogé. Toutefois, cette commission reste en fonctions jusqu'à l'installation de la Commission nationale de médecine et de biologie de la reproduction et du diagnostic prénatal instituée à l'article L. 184-3 du code de la santé publique. 
Décret $n^{\circ}$ 95-560 du 6 mai 1995 relatif aux activités d'assistance médicale à la procréation et modifiant le code de la santé publique (deuxième partie : Décrets en Conseil d'Etat) (J.O. du 7 mai 1995, 7363-7366)

Art. 1er. - 1.- Est inséré dans le code de la santé publique (deuxième partie : Décrets en Conseil d'Etat) un livre II intitulé : "Action sanitaire et médico-sociale en faveur de la famille, de l'enfance et de la jeunesse». le».

II. - Est inséré dans ce livre II un titre Ier intitulé : «Protection maternelle et infanti-

Art.2. - Est inséré au sein de ce titre Ier un chapitre II bis ainsi rédigé :

«Chapitre II bis

\section{"Assistance médicale à la procréation}

«Art. R. 152-9-1. - Les activités d'assistance médicale à la procréation mentionnées à l'article L.152-9 comprennent :

$\ll 1^{\circ}$ Les activités cliniques suivantes :

«a) Recueil par ponction d'ovocytes ;

«b) Recueil par ponction de spermatozoïdes ;

«c) Transfert des embryons en vue de leur implantation ;

« $2^{\circ}$ Les activités biologiques suivantes :

«a) Recueil et traitement du sperme en vue d'une assistance médicale à la procréation;

«b) Traitement des ovocytes ;

«c) Fécondation in vitro sans micro-manipulation;

«d) Fécondation in vitro par micro-manipulation;

«e) Conservation des gamètes ;

«f) Conservation des embryons en vue de transfert.

«Art. R. 152-9-2. - Les praticiens sous la responsabilité desquels sont effectuées les activités cliniques ou biologiques définies à l'article $R$ 152-9-1 doivent être, conformément à l'article L. 152-9, nommément agréés pour une ou plusieurs de ces activités ; l'agrément est donné par arrêté du ministre chargé de la santé pris après avis de la commission nationale de médecine et de biologie de la reproduction et du diagnostic prénatal.

«Pour chaque activité au titre de laquelle un praticien est agréé, l'agrément indique l'établissement dans lequel ledit praticien exercera cette responsabilité ; cet établissement doit avoir l'autorisation prévue à l'article L. 184-1 ou à l'article L. 673-5.

«Art. R. 152-9-3. - Lle praticien agréé au titre des activités définies au a et au c du $1^{\circ}$ de l'article R. 152-9-1 doit être médecin qualifié en gynécologie-obstétrique, avoir suivi une formation en médecine de la reproduction et justifier d'une expérience en médecine de la reproduction.

«Le praticien agréé pour effectuer les activités définies au b du $1^{\circ}$ de l'article R. 152-91 doit être médecin qualifié en urologie ou en chirurgie générale ou en gynécologie-obstétrique. Dans tous les cas, l'intéressé doit avoir acquis une formation ou une expérience dans le domaine de l'andrologie (souligné par la rédaction). 
«Art. R.152-9-4. - Le praticien pour effectuer les activités définies au $2^{\circ}$ de l'article R. 152-9-1 doit être médecin qualifié en biologie médicale, ou pharmacien biologiste, ou, à défaut, être une personnalité scientifique justifiant d'une formation particulière en biologie de la reproduction.

«Ce praticien doit être titulaire d'un certificat de biologie de la reproduction ou, à défaut, de titres jugés suffisants.

«Dans tous les cas, l'intéressé doit posséder une expérience suffisante dans la manipulation des gamètes humains.

«Art. R. 152-9-5. - Tout praticien agréé en application des articles L. 152-9 et R. 152-92 pour l'exercice d'activités dans un laboratoire d'analyses de biologie médicale doit en être le directeur ou un directeur adjoint.

«Art. R. 152-9-6. - L'agrément du praticien prend fin à l'expiration de la période de cinq ans, prévue au cinquième alinéa de l'article L. 184-1 et au troisième alinéa de l'article L. 673-5, pour laquelle l'autorisation a été délivrée à l'établissement.

«Art. R. 152-9-7. - Le retrait de l'agrément du praticien est encouru en cas de violation des prescriptions législatives et réglementaires applicables à l'assistance médicale à la procréation ainsi qu'en cas de violations des conditions fixées par l'agrément.

"La décision de retrait est prise après avis motivé de la commission nationale de médecine et de biologie de la reproduction et du diagnostic prénatal. Le praticien est invité à présenter ses observations devant la commission.»

«Art. 3. - I. - Est inséré dans le titre Ier du livre II du code de la santé publique (deuxième partie : Décrets en Conseil d'Etat) un chapitre V intitulé : «Du contrôle de certains établissements».

II. - Est inséré dans ce chapitre V une section 4 ainsi rédigée : «Section 4

«Activités d'assistance médicale à la procréation

«Sous-section I

«Conditions d'autorisation et de fonctionnement des activités d'assistance médicale à la procréation

«Paragraphe I

\section{«Dispositions générales}

«Art. R. 184-1-1. - Sans préjudice des conditions définies aux $\mathrm{I}^{\circ}$ et $2^{\circ}$ de l'article L. 712-9, l'octroi ou le renouvellement de l'autorisation mentionnée à l'article L. 184-1, de pratiquer une ou plusieurs des activités cliniques ou biologiques d'assistance médicale à la procréation définies à l'article R. 152-9-1, à l'exclusion du recueil, du traitement et de la conservation des gamètes issus d'un don, est subordonné au respect des règles de fonctionnement fixées dans la présente sous-section en application du quatrième alinéa de l'article L. 184-1. Ces règles constituent les conditions techniques de fonctionnement mentionnées au $3^{\circ}$ de l'article L. 712-9.

«Cette autorisation est délivrée à un établissement de santé ou un laboratoire d'analyses de biologie médicale par arrêté du ministre chargé de la santé, pris dans les conditions fixées par l'article L. 184-1. 
«Lorsqu'un établissement de santé ou un laboratoire de biologie médicale comporte plusieurs sites, l'autorisation précise le ou les sites d'exercice de la ou des activités.

«Art. R. 184-1-2. - Les dispositions prévues aux articles R. 712-38 à 712-51 pour les autorisations d'activités de soins délivrées par le ministre chargé de la santé sont applicables aux demandes d'autorisation et de renouvellement d'autorisation prévues par la présente sous-section.

«Toutefois, les pièces du dossier justificatif prévu à l'article R. 712-40 sont complétées ou remplacées par les pièces d'un dossier spécifique dont la composition est fixée par arrêté du ministre chargé de la santé.

«Art. R. 184-1-3. - La forme, la périodicité et le contenu de l'évaluation périodique des activités cliniques et biologiques d'assistance médicale à la procréation sont définis par arrêté du ministre chargé de la santé.

«Paragraphe 2

\section{«Conditions de fonctionnement des établissements de santé pratiquant les activi- tés cliniques d'assistance médicale à la procréation}

«Art. 184-1-4. - L'établissement de santé dans lequel sont pratiquées les activités définies au $1^{\circ}$ de l'article R.152-9-1 doit disposer d'un médecin expérimenté en échographie et d'un anesthésiste réanimateur. En outre, l'établissement doit s'assurer le concours d'un psychologue ou d'un médecin qualifié en psychiatrie.

«Art. R. 184-1-5. - Les activités définies aux a et c du $1^{\circ}$ de l'article R. 152-9-1 doivent être exercées au sein d'une structure de gynécologie ou de gynécologie-obstétrique, dans des locaux comprenant une pièce pour les entretiens des couples avec l'équipe médicale, prévus à l'article L.152-10, une salle de ponction équipée d'un échographe de haute définition avec sonde vaginale, un bloc opératoire, une salle de réveil et des lits d'hospitalisation.

«Les activités définies au b du $1^{\circ}$ de l'article R. 152-9-1 sont réalisées dans une structure chirurgicale.

«Art. R. 184-1-6. - L'établissement de santé doit respecter les règles de bonnes pratiques définies par arrêté du ministre chargé de la santé.

«Art. R. 184-1-7. - L'établissement de santé doit conserver dans le respect de la confidentialité :

$1^{\circ}$ La copie des pièces attestant du respect des conditions prévues au troisième alinéa de l'article L. 152-2 ;

$\left.2^{\circ}\right)$ Le consentement écrit du couple bénéficiaire de l'assistance médicale à la procréation, formulé avant la mise en œuvre de celle-ci et avant le transfert de l'embryon ou avant l'insémination, ainsi que, dans le cas où l'intervention d'un tiers donneur est nécessaire, la mention de la date et du lieu de la déclaration conjointe du couple prévue aux articles 31120 du code civil et 1157-2 du nouveau code de procédure civile.

"Art. R. 184-1-8. - L'établissement de santé doit conserver également dans le respect de la confidentialité, pour chaque couple concerné : 
« $1^{\circ}$ L'indication médicale de la mise en cuvre de l'assistance médicale à la procréation;

$\ll 2^{\circ}$ La date des ponctions de gamètes et le nombre d'ovocytes recueillis lors de chacune des ponctions ;

$« 3^{\circ}$ La date des transferts et le nombre d'embryons transférés ;

« $4^{\circ}$ Toute information disponible relative à l'évolution des grossesses et à l'état de santé des nouveau-nés.

«Paragraphe 3

«Conditions de fonctionnement des établissements de santé et des laboratoires pratiquant les activités biologiques d'assistance médicale à la procréation

«Art. R. 184-1-9. - L'établissement de santé ou le laboratoire d'analyses de biologie médicale dans lequel sont pratiqués les activités définies au $2^{\circ}$ de l'article R. 151-9-1 doit comprendre une pièce exclusivement affectée au recueil du sperme, une pièce exclusivement affectée au traitement des gamètes et à la fécondation in vitro et une pièce exclusivement affectée à la conservation des gamètes et des embryons. Il doit disposer en outre de l'équipement et du matériel nécessaires à la mise en œuvre de ces activités et doit être en mesure d'en assurer la décontamination et la stérilisation.

"La pièce affectée à la conservation des gamètes et des embryons doit être équipée d'une protection contre le vol.

«Pour les activités mentionnées aux c et $\mathrm{d}$ du $2^{\circ}$ de l'article $R .152-9-1$, la pièce affectée au traitement des gamètes et à la fécondation in vitro doit être équipée d'une hotte à flux laminaire ou d'un matériel équivalent.

«Pour l'activité visée au d du $2^{\circ}$ de l'article R. 152-9-1, l'établissement ou le laboratoire doit disposer d'un matéreil spécifique adapté aux micromanipulations.

«Art. R. 184-1-10. - Lorsque les activités définies au $2^{\circ}$ de l'article R. 152-9-1 sont pratiquées par un laboratoire d'analyses de biologie médicale, elles peuvent être effectuées, en application de l'article 8 du décret $n^{\circ} 76-1004$ du 4 novembre 1976 , dans un local distinct de celui du laboratoire. ce local doit alors répondre aux conditions prévues à l'article R. 184-1-9.

«Art. R. 184-1-11. - L'établissement de santé ou le laboratoire doit respecter les règles de bonnes pratiques définies par arrêté du ministre chargé de la santé.

"Art. R. 184-1-12. - L'établissement de santé ou le laboratoire conserve, dans le respect de la confidentialité, pour chaque couple et chaque tentative d'assistance médicale à la procréation, la mention:

« $1^{\circ} \mathrm{Du}$ nombre d'ovocytes traités ;

$\ll 2^{\circ}$ De la date de fécondation;

$« 3^{\circ}$ Du nombre d'embryons obtenus.

«Paragraphe 4

«Obligations des établissements de santé et des laboratoires en ce qui concerne la conservation des gamètes ou des embryons

«Art. R. 184-1-13. - Aucune interruption ou cessation d'activité d'un établissement ou d'un laboratoire autorisé à conserver les gamètes ou les embryons ne doit avoir pour conséquence l'arrêt de la conservation de ceux-ci. 
«A cette fin, tout établissement de santé ou tout laboratoire autorisé à conserver des gamètes ou des embryons doit passer un accord avec un autre établissement ou laboratoire autorisé à pratiquer la même activité, en vue du déplacement éventuel des gamètes ou des embryons. Cet accord doit être transmis au ministre chargé de la santé dans un délai de six mois à compter de la notification de l'autorisation ou de son renouvellement.

«Tout déplacement de gamètes ou d'embryons, doit être signalé préalablement au ministre chargé de la santé. Dans le cas où il ne s'effectuerait pas conformément à l'accord prévu au précédent alinéa, il devrait être autorisé à pratiquer la même activité pour recevoir les gamètes ou les embryons.

«Art. R. 184-1-14. - Toute personne ayant consenti à la conservation des gamètes ou des embryons, à l'exclusion des donneurs de gamètes, doit être préalablement informée de leur déplacement ainsi que du nouveau lieu de conservation.

«Art. R. 184-1-15. - Les registres de gamètes et d'embryons décrits à l'article R. 184-21 et R. 184-2-2 ainsi que, le cas échéant, les informations mentionnées à l'article R. 673-5-8 concernant le donneur de gamètes doivent être transmis à l'établissement de santé ou du laboratoire accueillant les gamètes ou les embryons dans des conditions garantissant la confidentialité.

«Paragraphe 5

\section{«Registres relatifs aux gamètes et aux embryons}

"Art. R.184-2-1. - Le registre des gamètes que doit tenir tout établissement de santé ou tout laboratoire autorisé à conserver des gamètes doit mentionner :

« $1^{\circ}$ Soit l'identité de la personne sur laquelle ont été recueillis les gamètes lorsqu'il s'agit d'une assistance médicale à la procréation réalisée sans le recours à un tiers donneur, soit le code d'identification du donneur ;

« $2^{\circ}$ Le lieu et les dates de congélation des gamètes;

$« 3^{\circ}$ Les dates et les modes d'utilisation des gamètes ;

$« 4^{\circ}$ Les indications précises du lieu de conservation des gamètes dans la pièce affectée à cet effet ;

« $5^{\circ}$ Le cas échéant, l'identité du couple destinaire du don de gamètes.

«Art. R. 184-2-2. - Le registre d'embryons que doit tenir tout établissement de santé ou tout laboratoire autorisé à conserver des embryons doit mentionner :

« $1^{\circ}$ L'identité du couple qui est à l'origine de l'embryon, et, le cas échéant, le code d'identification du donneur de gamètes ;

« $2^{\circ}$ Le nombre d'embryons conservés pour chaque couple ;

$« 3^{\circ}$ Le lieu et les dates de fécondation et de congélation;

« $4^{\circ}$ Le cas échéant, le lieu de conservation antérieure ;

$\ll 5^{\circ}$ Les indications précises du lieu de conservation des embryons dans la pièce affectée à cet effet ;

$« 6^{\circ}$ Les informations relatives au devenir de chaque embryon, notamment la date de décongélation.

«Art. R.184-2-3. - Les praticiens agréés pour la conservation des gamètes ou pour la conservation des embryons doivent veiller à la bonne tenue des registres mentionnés aux 
articles R.184-2-1 et R. 184-2-2 et à l'exactitude des informations qui y sont consignées.

«Art. R.184-2-4. - Ces registres doivent être reliés et numérotés et gardés dans des locaux situés à proximité de ceux où sont conservés les gamètes ou les embryons, dans des conditions garantissant la confidentialité.»

«Art. 4. - I. - Est inséré dans le livre VI du code de la santé publique (deuxième partie : Décrets en Conseil d'Etat), au titre III, un chapitre II intitulé : «Des tissus, cellules et produits".

II. - Est insérée dans ce chapitre II une section V ainsi rédigée :

\section{«Section V}

\section{«Dispositions spécifiques au don et à l'utilisation de gamètes}

"Art. R. 673-5-1. - Sans préjudice des conditions définies aux $1^{\circ}$ et $2^{\circ}$ de l'article L. 712-9, l'octroi ou le renouvellement de l'autorisation mentionnée à l'article L.673-5, nécessaire aux organismes sans but lucratif et aux établissements de santé publics et privés à but non lucratif pour pratiquer une ou plusieurs des activités de recueil, traitement, conservation et cession de gamètes issus d'un don, est subordonné aux règles fixées par la présente section en application du deuxième alinéa de l'article L. 673-5. Ces règles constituent les règles techniques de fonctionnement mentionnées au $3^{\circ}$ de l'article L. 712-9.

«Cette autorisation est délivrée par arrêté du ministre chargé de la santé dans les conditions fixées par l'article L. 673-5.

«Lorqu'un organisme ou un établissement de santé comporte plusieurs sites, l'autorisation précise le ou les sites d'exercie de la ou des activités.

«Art. R. 673-5-2. - Les dispositions prévues aux articles R. 712-38 à R. 712-51 pour les autorisations d'activités de soins délivrées par le ministre chargé de la santé sont applicables aux demandes d'autorisation et de renouvellement d'autorisation prévues par la présente section.

«Toutefois, les pièces du dossier justificatif prévu à l'article R. 712-40 sont complétées ou remplacées par les pièces d'un dossier spécifique dont la composition est fixée par arrêté du ministre chargé de la santé.

«Art. R. 673-5-3. - Sont applicables aux organismes ou établissements mentionnés à l'article R. 673-5-1 les dispositions de l'article R. 184-1-3, les dispositions des paragraphes 2 et 3 de la sous-section I de la section 4 du chapitre $\mathrm{V}$ du livre II en tant qu'elles concernent le recueil, le traitement et la conservation des gamètes, les dispositions du paragraphe 4 de la même sous-section en tant qu'elles concernent la conservation des gamètes, et les dispositions du paragraphe 5 de la même sous- section en tant qu'elles concernent les registres de gamètes.

«Art. R. 673-5-4. - La disposition des locaux ainsi que les modalités d'accueil des donneurs et des couples receveurs doivent être de nature à assurer l'anonymat du don et la confidentialité des activités.

«Une pièce est aménagée pour les entretiens préalables au don ou à la cession de gamètes.

«Art. R. 673-5-5. - Le consentement du donneur et celui de l'autre membre du couple 
prévus à l'article L. 673-2 ainsi que le receuil des gamètes doivent être précédés d'entretiens entre le donneur et les membres de l'équipe médicale ayant pour but notamment :

« $1^{\circ}$ De vérifier que le donneur remplit les conditions prévues à l'article L. 673-2 ;

« $2^{\circ}$ De l'informer des dispositions législatives et réglementaires relatives au don de gamètes et de leurs conséquences au regard de la filiation ;

$« 3^{\circ}$ De lui préciser la nature des examens à effectuer avant le don;

« $4^{\circ}$ De lui indiquer qu'il devra consentir à la conservation dans le dossier mentionné à l'article R. 673-5-8 d'informations à caractères personnels relatives à sa santé.

«Art. R.673-5-6. - Sans préjudice des dispositions de l'article L. 152-10, toute cession de gamète doit être précédée d'un ou plusieurs entretiens du couple destinataire du don avec une équipe médicale pluridisciplinaire à laquelle doit s'adjoindre un médecin qualifié en psychiatrie ou un psychologue.

«Art. R.673-5-7. - Les gamètes provenant d'un don ne peuvent être cédés qu'au praticien réalisant l'assistance médicale à la procréation ou au couple destinataire du don.

«Art. R. 673-5-8. - Pour remplir les obligations prévues à l'article L. 673-6, les organismes et établissements de santés autorisés pour les activités de recueil, traitement, conservation et cession de gamètes issus d'un don conservent des informations sur le donneur :

«Le dossier du donneur contient, sous forme rendue anonyme :

« $1^{\circ}$ Les antécédents médicaux personnels et familiaux nécessaires à la mise en œuvre de l'assistance médicale à la procréation avec tiers donneur ;

« $2^{\circ}$ Les résultats de tests de dépistage sanitaires obligatoires ;

$\ll 3^{\circ}$ Le nombre d'enfants issus du don;

" $4^{\circ}$ S'il s'agit d'un don de sperme, la date des dons, le nombre de paillettes conservées, la date des cessions et le nombre de paillettes cédées ;

«5 ${ }^{\circ}$ S'il s'agit d'un don d'ovocyte, la date de la ponction et le nombre d'ovocytes donnés ;

$« 6^{\circ}$ Le consentement écrit du couple auquel appartient le donneur.

"Les praticiens agréés pour les activités mentionnées au premier alinéa, conformément à l'article L. 152-9 et à l'article R. 152-9-2, sont responsables de la bonne tenue du dossier et de l'exactitude des informations qui y sont consignées.

«Ce dossier doit être conservé quel que soit son support sous forme anonyme. L'archivage doit être effectué dans des conditions garantissant la confidentialité.

"Le donneur doit, avant le recueil des gamètes, donner expressément son consentement à la conservation de ce dossier.

"Les informations touchant à l'identité des donneurs, à l'identification des enfants nés et aux liens biologiques existant entre eux sont conservées dans une chambre ou armoire forte spécifiquement affectée à cette conservation à laquelle ont accès uniquement les praticiens agréés pour les activités mentionnées au premier alinéa.

«Art. R. 673-5-9. - En vue de se conformer aux prescriptions de l'article L. 673-4 et pour permettre l'accès aux informations médicales dans les conditions prévues à la deuxième phrase de l'article L. 673-6, l'établissement ou l'organisme conserve toute information relative à l'évolution des grossesses induites par un don de gamètes, y compris leur éventuelle interruption, la date de naissance et l'état de santé des nouveau-nés.»

«Art. 5. - Le 11 du III de l'article R. 712-2 du code de la santé publique est remplacé par les dispositions suivantes : 
"11 Activités cliniques d'assistance médicale à la procréation, activités biologiques d'assistance médicale à la procréation, activités de recueil, traitement, conservation de gamètes et cession de gamètes issus de don, activités de diagnostic prénatal.»

«Art. 6. - les autorisations délivrées en application du décret nº88-327 du 8 avril 1988 cessent d'être valables six mois après la publication du présent décret si, dans ce délai, les établissements de santé, les laboratoires d'analyses de biologie médicale ou les organismes concernés n'ont pas demandé l'autorisation ou les autorisations dont relèvent leurs activités en fournissant aux autorités compétentes le dossier prévu aux articles R.712-40, R.1841-2 et R.673-5-2 du code de la santé publique.

Par dérogation à l'article R. 712-39 du même code, les autres établissements, laboratoires ou organismes peuvent également déposer un dossier de demande d'autorisation dans la période de six mois courant à compter de la publication du présent décret.

L'expiration de la période mentionnée aux alinéas précédents fait courir le délai de six mois prévu au troisième alinéa de l'article L. 712-16 du même code.

«Art. 7. - Le décret $\mathrm{n}^{\circ} 88.327$ du 8 avril 1988 relatif aux activités de procréation médicalement assistée est abrogé. 
Arrêté du 2 juin 1995 fixant la composition du dossier prévu aux articles R.184-12 et R. 673-5-2 du code de la santé publique à produire à l'appui d'une demande d'autorisation de pratiquer des activités d'assistance médicale à la procréation. (J.O. du 10 juin 1995, 8994)

Art.1er. - Les établissements de santé, les laboratoires d'analyses de biologie médicale et les organismes sans but lucratif désirant pratiquer une ou plusieurs des activités d'assistance médicale à la procréation définies à l'article R. 152-9-1 du code de la santé publique doivent produire à l'appui de leur demande, outre le dossier justificatif prévu à l'article $R$. 712-40 du code de la santé publique, le dossier spécifique mentionné aux articles R. 184-1-2 et R. 673-5-2 dont le contenu est fixé au présent arrêté (1).

Art. 2. - Le contenu du dossier concernant les activités cliniques d'assistance médicale à la procréation définies au $1^{\circ}$ de l'article R. 159-9-1 du code de la santé publique est fixé à l'annexe I du présent arrêté.

Art. 3. - Le contenu du dossier concernant les activités biologiques d'assistance médicale à la procréation définies au $2^{\circ}$ de l'article R. 152-9-1 du code de la santé publique est fixé à l'annexe II du présent arrêté.

Art. 4. - Le dossier dont le contenu est fixé aux annexes I et II du présent arrêté doit être accompagné, pour chaque responsable proposé pour l'agrément, et dans le domaine de la médecine ou de la biologie de la reproduction :

-d'un curriculum vitae ;

-de la copie des diplômes, titres, certificats ;

-des attestations des stages effectués.

Art. 5. - L'ensemble du dossier est transmis par le demandeur en cinq exemplaires au directeur départemental des affaires sanitaires et sociales du département où se trouve l'établissement, le laboratoire d'analyses de biologie médicale ou l'organisme sans but lucratif.

(1) L'arrêté, accompagné de ses annexes, sera publiqué intégralement au Bulletin officiel du ministère de la santé publique et de l'assurance maladie $\mathrm{n}^{\circ} 95-25$, disponible à la Direction des Journaux officiels, 26, rue Desaix, 75727 Paris Cedex 15, au prix de 31,50F. 